\title{
Expert review on systemic treatment in the St. Gallen International Breast Cancer Conference 2021
}

\author{
Mingxi Lin ${ }^{1,2 \#}$, Yizi Jin ${ }^{1,2 \#}$, Jian Zhang ${ }^{1,2}$ \\ ${ }^{1}$ Department of Medical Oncology, Fudan University Shanghai Cancer Center, Shanghai, China; ${ }^{2}$ Department of Oncology, Shanghai Medical \\ College, Fudan University, Shanghai, China \\ Contributions: (I) Conception and design: J Zhang; (II) Administrative support: J Zhang; (III) Provision of study materials or patients: J Zhang; (IV) \\ Collection and assembly of data: M Lin, Y Jin; (V) Data analysis and interpretation: M Lin, Y Jin; (VI) Manuscript writing: All authors; (VII) Final \\ approval of manuscript: All authors. \\ "These authors contributed equally to this work. \\ Correspondence to: Jian Zhang. Department of Medical Oncology, Fudan University Shanghai Cancer Center, Shanghai, China; Department of \\ Oncology, Shanghai Medical College, Fudan University, No. 270, Dong'an Road, Shanghai 200032, China. Email: syner2000@163.com.
}

\begin{abstract}
The $17^{\text {th }}$ St. Gallen International Breast Cancer Consensus Conference was held in March 2021. The theme of this year's conference was “customizing local and systemic therapies". The conference focused on the controversies about the optimal primary treatment of early breast cancer. The St. Gallen expert panel reviewed substantial new evidences and voiced their expert opinions, hoping to provide guidance to clinicians. Despite that many randomized clinical trials provided evidence on managing breast cancer, not all the clinical scenarios can find definitive data to guide recommendations. When data from randomized clinical trials are lacking, the opinions of the St. Gallen expert panel can be used as an important reference for clinical decision making. However, the panelists' recommendations may often be affected by the treatment availability, which vary from country to country. As a result, from Chinese experts' perspectives, Professor Jian Zhang made an interpretation and comments of the expert opinions of the $17^{\text {th }}$ St. Gallen Conference. This work focused on the field of systemic treatment, including neoadjuvant therapy (chemotherapy, HER2 targeted therapy and endocrine therapy), tailored post-neoadjuvant therapy based on neoadjuvant therapy response and adjuvant therapy. We hope this work could help clinicians understand the expert opinions of the $17^{\text {th }}$ St. Gallen Conference better.
\end{abstract}

Keywords: St. Gallen; neoadjuvant therapy; adjuvant therapy; early breast cancer

Received: 06 June 2021; Accepted: 28 July 2021; Published: 31 July 2021.

doi: $10.21037 /$ tbcr-21-7

View this article at: https://dx.doi.org/10.21037/tbcr-21-7

\section{Introduction}

The $17^{\text {th }}$ St. Gallen International Breast Cancer Consensus Conference was held in March 2021. This conference is a multidisciplinary and global meeting. Experts, who actively engaged in clinical or basic research of breast cancer, constituted the Consensus Panel. 74 panelists participated and voted in this conference (1). This conference focused on the controversies about the optimal primary treatment of early breast cancer, including the fields of genetics, pathology, imaging, neoadjuvant/adjuvant therapy, surgery, and radiation therapy (2). The goal of the meeting was to discuss the practical issues in the management of breast cancer, and provide guidance to clinicians. The St. Gallen expert panel agreed that data from randomized clinical trials had the highest level of evidence. However, when data are lacking, the opinions of the St. Gallen expert panel can be used as an important reference for clinical decision making. From Chinese experts' perspectives, Professor Jian Zhang made an interpretation and comment of the discussion results of the $17^{\text {th }}$ St. Gallen International Breast Cancer Consensus Conference in the field of systemic treatment. 


\section{Neoadjuvant therapy}

\section{Neoadjuvant therapy for HR+/HER2- breast cancer}

For postmenopausal women with low-risk genomic signature or low-grade breast cancer, if neoadjuvant therapy is needed, there was no superiority for neoadjuvant chemotherapy over neoadjuvant endocrine therapy: (I) agreed: $98.2 \%$; (II) disagreed: $1.8 \%$ (abstain: 3 ).

HR+/HER2 - breast cancer had lower response rates for neoadjuvant chemotherapy than other biological subtypes. When neoadjuvant therapy is needed (for example, to downstage breast cancer), neoadjuvant endocrine therapy may be a low-toxicity alternative option. Semiglazov et al. compared neoadjuvant chemotherapy (doxorubicin with paclitaxel) with neoadjuvant endocrine therapy (anastrozle or exemestane) in HR+ breast cancer patients (3). Clinical objective response (both 64\%) and rates of pathological complete response $(6 \%$ vs. $3 \%)$ were similar in both treatment groups. A meta-analysis (4) included over 20 studies also showed that neoadjuvant endocrine therapy gained similar response rates to neoadjuvant chemotherapy. TransNEOS trial (5) included 295 patients with $\mathrm{HR}+$ / HER2- breast cancer, and 21-gene recurrence score (RS) test was performed before neoadjuvant endocrine therapy. The response rates were $54 \%$ for patients with $\mathrm{RS}<18$, but only $22 \%$ for RS $>31$. The American College of Surgeons Oncology Group (ACOSOG) Z1031 study confirmed that after 16-18 weeks of neoadjuvant endocrine therapy (aromatase inhibitor), half patients could undergo successful breast conserving surgery (6). 2021 ASCO guideline recommended that neoadjuvant endocrine therapy with aromatase inhibitor could be given to postmenopausal patients with $\mathrm{HR}+$ breast cancer (7).

However, in China, the adoption of neoadjuvant endocrine therapy into clinical practice is still slow. This is probably because the pathological complete response (pCR) rates of neoadjuvant endocrine therapy are low, and this endpoint lacks of significantly prognostic value. Moreover, 21-gene test has not been verified by large samples in China.

Patients with ER+ breast cancer and residual disease after neoadjuvant endocrine therapy should be offered adjuvant chemotherapy: (I) if the patient has excellent clinical response with node-negative residual cancer? (i) Agreed: $0.0 \%$, (ii) disagreed: $100.0 \%$ (abstain: 3 ); (II) if the patient has residual positive lymph nodes? (i) Agreed: $52.9 \%$, (ii) disagreed: $47.1 \%$ (abstain:7); (III) if the patient has $>5 \mathrm{~cm}$ residual tumor? (i) Agreed: $77.2 \%$, (ii) disagreed: $22.9 \%$ (abstain: 1).
The neoadjuvant endocrine therapy could not only improve surgical outcome but also be used to adjust adjuvant treatment therapy. Ellis et al. utilized preoperative endocrine prognostic index (PEPI) to evaluate tumor response to neoadjuvant endocrine therapy (8). PEPI score 0 represented pT1/pT2, N0, Ki-67 $\leq 2.7 \%$, and ER Allred score $>2$ measured on surgical specimen after neoadjuvant endocrine therapy. They found patients with PEPI score 0 had extremely low risk of relapse. The ACOSOG Z1031B also used PEPI score to examine the risk of relapse (9). For patients with PEPI score 0, the 5-year relapse risk was only $3.6 \%$ without adjuvant chemotherapy, supporting omitting adjuvant chemotherapy in this group. Moreover, the prospective WSG-ADAPT HR+/HER2- trial (10) used biomarkers (RS scores and Ki-67 index) to assess which patient should receive adjuvant chemotherapy after 3 -week neoadjuvant endocrine therapy. In this study, patients with RS $0-11$ or RS $12-25$ plus Ki-67 $\leq 10 \%$ (assessed after 3-week neoadjuvant endocrine therapy) were spared adjuvant chemotherapy. As a result, de-escalation of therapy with no chemotherapy may be recommended for patients with excellent clinical response after neoadjuvant endocrine therapy. On the other hand, residual positive lymph nodes or $>5 \mathrm{~cm}$ residual tumor might represent lower endocrine sensitivity, and adjuvant chemotherapies are needed.

\section{Neoadjuvant therapy for HER2-positive breast cancer}

For HER2-positive breast cancer with clinically positive lymph nodes, the preferred neoadjuvant regimen should include taxane and trastuzumab, combined with: (I) anthracycline: $61.8 \%$; (II) pertuzumab: $12.7 \%$; (III) pertuzumab plus platinum: $23.6 \%$ (abstain: 1 ).

In the era of effective anti-HER 2 therapies, the contribution of anthracyclines has always been controversial. Whether the addition of anthracyclines in neoadjuvant therapy would offer benefit in HER2positive breast cancer remains unclear. TRAIN-2 $(11,12)$ and TRYPHAENA (13) were the two key trials addressing this question. The TRAIN-2 directly compared the 9 cycles of anthracycline-free regimen [paclitaxel + carboplatin + trastuzumab + pertuzumab (PCbHP)] with the anthracycline-containing regimen [5-fluorouracil + epirubicin + cyclophosphamide (FEC) + HP for 3 cycles followed by PCbHP for 6 cycles]. The study found that the addition of anthracycline did not improve pCR rates or event-free survival (EFS) in neoadjuvant therapy. However, the 9-cycle treatment regimen was not the 
standard therapy. The TRYPHAENA trail also compared the 6-cycle anthracycline-containing regimen (FEC + HP for 3 cycles followed by docetaxel[T]+HP for 3 cycles) and the 6-cycle anthracycline-free regimen (TCbHP). The two treatment arms had similar pCR rates, corroborating the results from TRAIN-2. However, the primary objective of TRYPHAENA was to evaluate tolerability and safety. The efficacy results should not be over-interpreted. As a result, more evidences are needed to support the elimination of anthracycline in neoadjuvant therapy.

For HER2-positive breast cancer patients with clinically positive lymph nodes, if the neoadjuvant chemotherapy and trastuzumab plus pertuzumab (HP) are given and the tumor achieves pCR, expert panel agreed that the preferred adjuvant anti-HER2 therapy is: (I) HP irrespective of baseline stage: 55.6\%; (II) HP if baseline stage 3: 22.2\%; (III) $\mathrm{H}$ alone: $22.2 \%$ (abstain: 5 ).

For HER2-positive patients with clinically negative lymph node, if neoadjuvant HP are given and the tumor chieves pCR, experts agreed that: (I) $\mathrm{H}$ alone would be enough: 69.6\%; (II) HP if baseline stage 1/2: 12.5\%; (III) HP if baseline stage 2: 17.9\% (abstain: 3).

For patients with a pCR after neoadjuvant chemotherapy, trastuzumab and pertuzumab, no data supported the administration of continued trastuzumab plus pertuzumab or trastuzumab alone, to complete the 1-year duration of anti-HER2 therapy. A pCR following neoadjuvant chemotherapy is often associated with improved prognosis. However, a small group of patients would still experience a relapse. It's of great interest to identify patients with high risk of relapse despite a $\mathrm{pCR}$ and propose the addition of pertuzumab beyond trastuzumab and chemotherapy. Huober $e t a l$. performed a pooled retrospective analysis to identify factors associated with relapse despite a pCR (14). 2,188 patients with pCR were included. The investigators found that the initial positive lymph node was significantly related with shorter disease-free survival $(\mathrm{cN}+v s$. $\mathrm{cN} 0$, $\mathrm{HR}=1.70,95 \% \mathrm{CI}: 1.2-2.4, \mathrm{P}=0.002)$. The 6 -year followup of APHINITY trial (15) demonstrated that patients with positive lymph nodes would benefit from adding pertuzumab to standard adjuvant therapy. The 6-year invasive disease-free survival was $83 \%$ with placebo and $88 \%$ with pertuzumab (HR $=0.72,95 \%$ CI: $0.59-0.87$ ). For patients with negative lymph nodes, no difference was seen. As a result, for patients with $\mathrm{cN}+$ and received $\mathrm{pCR}$ after neoadjuvant therapy, the addition of pertuzumab is recommended. The seven-year follow-up of the APT trial (16) suggested that patients with $\mathrm{N}$ - and small
HER2-positive breast cancer had minimal risk of disease recurrence. The seven-year disease-free survival of adjuvant paclitaxel and trastuzumab was 93.3\% (95\% CI: 90.4-96.2). As a result, for patients with $\mathrm{cN}$ - breast cancer and $\mathrm{pCR}$ after neoadjuvant therapy, trastuzumab alone would be enough.

\section{Neoadjuvant therapy for triple negative breast cancer (TNBC)}

For patients with TNBC, if standard neoadjuvant chemotherapy is given, an immune checkpoint inhibitor is not needed: (I) agreed: 90.8\%; (I) disagreed: 9.6\% (abstain: 4).

Although Keynote522 (17) and IMpassion031 (18) trials suggested that the addition of immune checkpoint inhibitors may augment pCR, the toxicity and long-term survival outcomes are still unclear. And immune checkpoint inhibitors targeting PD1/PDL1 have not gained indications in China. The biomarker for clinical benefit of immune checkpoint inhibitor was not definite. We do not regularly recommend the addition of immune checkpoint inhibitors in neoadjuvant therapy for patients with TNBC.

PD1/PDL1 testing should not affect the recommendation for the use of immune checkpoint inhibitor in neoadjuvant therapy: (I) agreed: $81.1 \%$; (II) disagreed: $18.9 \%$; (abstain: 6).

The Keynote522 trial indicated that the addition of pembrolizumab in neoadjuvant therapy could increase the percentage of patients with pCR (17). The benefits were observed in both PD-L1-positive population and PD-L1negative population. The similar results were observed in IMpassion031 trial (18). As a result, the PD1/PDL1 testing should not affect the recommendation for the use of immune checkpoint inhibitor in neoadjuvant therapy.

All the patients with TNBC and residual disease after neoadjuvant therapy should receive adjuvant capecitabine: (I) agreed: $87.7 \%$; (I) disagreed: $12.3 \%$ (abstain: 1 ).

TNBC has high relapse rate, especially for patients with residual disease after neoadjuvant therapy. The CREATE-X trial evaluated the value of adjuvant capecitabine in patients with residual disease after standard neoadjuvant therapy (taxane, anthracycline, or both) (19). The study revealed that adjuvant capecitabine could increase the rate of disease-free survival $(74.1 \%$ in capecitabine group vs. $67.6 \%$ in control group, $\mathrm{HR}=0.70,95 \% \mathrm{CI}: 0.53-0.92$ ). Among patients with TNBC, the capecitabine also increased the rate of diseasefree survival $(69.8 \%$ in capecitabine group vs. $56.1 \%$ in 
control group, HR $=0.58,95 \%$ CI: $0.39-0.87)$ and the overall survival rate $(78.8 \%$ vs. $70.3 \%, \mathrm{HR}=0.52,95 \% \mathrm{CI}$ : $0.30-0.90)$. As a result, if the toxicities were tolerable, we recommended adjuvant capecitabine for TNBC that did not gain pCR after neoadjuvant therapy. Moreover, CBCSG010 trial (20) showed that the addition of capecitabine to standard adjuvant therapy could improve 5 -year diseasefree survival rates in TNBC patients $(86.3 \%$ vs. $80.4 \%$, $\mathrm{HR}=0.66$, 95\% CI: 0.44-0.99). And SYSUCC-001 trial (21) demonstrated the efficacy of maintenance therapy with low-dose capecitabine after standard adjuvant therapy for TNBC patients.

\section{Adjuvant therapy}

\section{Adjuvant therapy for HR-positive/HER2-negative breast cancer}

\section{ER threshold for endocrine therapy}

The appropriate threshold for "positive" ER expression for recommending adjuvant endocrine therapy in ER-positive breast cancers tested by IHC is: (I) greater than or equal to $1 \%$ : $50.0 \%$; (II) greater than or equal to $10 \%: 50.0 \%$ (abstain: 3).

According to the most updated ASCO/CAP guideline for ER/PR testing, breast cancers with $1-10 \%$ cells staining ER positive have been classified into a new category of ER low positive (22). Due to the relative rarity of this subpopulation, the evidence on the endocrine therapy benefits for breast cancers with ER low positive remained limited, and no clinical trial has so far been designed to address this question. Previous retrospective studies demonstrated inconsistent results regarding the prognosis and endocrine responsiveness of ER low positive breast cancers. Several studies suggested that the biological behavior and clinical outcome of ER low positive tumors were more similar to the ER-negative and gained limited benefits from adjuvant endocrine therapy (23-25). A real-world data analysis of 17,216 patients in China also confirmed these findings. ER low positive breast cancer tended to have strongly aggressive clinicopathological features and a majority of them were treated with chemotherapy (26). However, there were still data from long-term follow up supporting patients with ER expression $\geq 1 \%$ may benefit from endocrine therapy (27).

We think the possible benefit of endocrine therapy in the ER-low-positive patients should not be denied based on the current evidence. Considering the relative low toxicity of endocrine therapy, we endorse that adjuvant endocrine therapy can be considered in patients with ER expression $\geq 1 \%$. In the clinical practice for this subpopulation, more clinicopathological factors (such as tumor size, stage, histopathological types, Ki-67 index, etc.) should be considered to decide the administration of endocrine therapy. For those who have positive lymph nodes, the IHC tests for involved lymph nodes can also provide clues for treatment decisions. The controversial evidence also indicated that prospective studies are needed to help determine the threshold.

\section{Extended endocrine therapy}

For node-positive ER-positive HER2-negative breast cancers, what is the optimal duration of the endocrine therapy? (I) 5 years: $11.3 \%$; (II) 7 to 8 years: $34.0 \%$; (III) 10 years: $52.8 \%$; (VI) indefinite: $1.9 \%$ (abstain: 1 ).

For premenopausal high-risk patients who received 5 years of OFS + Tam, do you prolong treatment? (I) Yes, additional 5 years of OFS + Tam: $4.1 \%$; (II) Yes, additional 5 years of Tam only: 44.9\%; (III) Yes, additional 5 years of $\mathrm{AI}$ (+ OFS if still premenopausal): $40.8 \%$; (IV) No: $10.2 \%$ (abstain: 7).

Prolonged adjuvant endocrine therapy should be considered in ER-positive breast cancer patients with high-risk factors (such as positive lymph nodes, grade 3, $>\mathrm{T} 2, \mathrm{Ki}-67>30 \%$, etc.) after 5 -year standard therapy if tolerable. It is also noteworthy that prolonged adjuvant AI treatment contributes greatly to the prevention of second breast cancers. Yet the optimal duration and treatment regimens for extended adjuvant endocrine therapy remained controversial. For postmenopausal patients, NSABP-B42 (28) trial showed that the extension of endocrine therapy with aromatase inhibitors (AI) for additional 5 years decreased the recurrence risk, while IDEAL (29) and ABCSG-16 (30) demonstrated that extension with AI for additional 2 to 2.5 years yielded comparable benefit compared with additional 5 years. For premenopausal patients, although 10-year tamoxifen treatment did not demonstrate significant improvement in OS for node-negative patients compared with the 5-year regimen in NSABP B-14 (31), the results from ATLAS (32) and aTTom (33) endorsed the 10-year tamoxifen therapy due to its benefit for risk reduction. For premenopausal patients who received 5 years of OFS + Tam/AI, although there lacks straightforward proof from clinical trial, we still consider the extension of endocrine therapy based on the evidence of decreasing long-term recurrence risk. The combined analysis of TEXT \& SOFT study demonstrated 
that patients with high risks benefit more from 5 year of OFS + AI compared with OFS + Tam in the long term (34). Therefore, if the premenopausal patient had higher risks such as having $\geq 4$ positive nodes, aged $\leq 35$, and/or grade 3 , additional 5 years of more escalated therapy, i.e., OFS+AI should be preferred. The side effect of long-term tamoxifen such as an increased risk of thrombosis and endometrial cancer should also be considered.

\section{CDK4/6 inhibitors}

Should patients with ER-positive cancers and 4 or more positive lymph nodes receive adjuvant CDK4/6 inhibitor therapy with abemaciclib? (I) Yes: $54.0 \%$; (II) No: $46.0 \%$ (abstain: 7).

Should patients with ER-positive cancers and 1-3 positive lymph nodes and with grade 3, or/and T3, or high $\mathrm{Ki}-67$ receive adjuvant CDK4/6 inhibitor therapy with abemaciclib (i.e., Monarch-E eligibility)? (I) Yes: 43.6\%; (II) No: $53.7 \%$ (abstain: 3 ).

In ER-positive, HER2-negative breast cancer, should Ki67 testing (in combination with other prognostic markers) be used to select adjuvant treatment with CDK 4/6 inhibitors? (I) Yes: 39.6\%; (II) No: 60.4\% (abstain: 4).

According to the interim analysis of Monarch-E, abemaciclib combined with endocrine therapy significantly improved iDFS $(\mathrm{P}=0.01, \mathrm{HR}=0.75,95 \% \mathrm{CI}: 0.60-0.93)$ and DRFS ( $\mathrm{P}=0.01, \mathrm{HR}=0.72,95 \% \mathrm{CI}$ : $0.56-0.92$ ) versus endocrine therapy alone (35). In the subgroup analysis stratified by lymph nodes, patients with 4-9 positive lymph nodes benefited the most (iDFS: HR $=0.69,95 \%$ CI: 0.48-0.99), which could serve as solid evidence supporting the use of abemaciclib in this subpopulation. For patients with 1-3 positive lymph nodes, the iDFS improvement was less significant (HR $=0.71,95 \%$ CI: 0.48-1.06). It should be noted that although the subgroup analyses were prespecified, the subgroup results were exploratory in nature due to statistical reasons.

Among the three adjuvant CDK inhibitor trials, Monarch-E was the only trial demonstrated positive results. Compared with Monarch-E, the PALLAS trial enrolled more patients with $\mathrm{N} 0$ or $\mathrm{N} 1$ pathological nodal status. The negative results of PALLAS (36) might be the reason why more clinicians were cautious on the addition of CDK4/6 inhibitor in patients with 1-3 positive lymph nodes and other high-risk factors. Another issue is how to determine the "high-risk" population. Anatomic stage mainly identifies patients with high risk of early relapse, while biological risk may predict recurrence risk in the long run. Apart from clinical risk, biological risk was also considered in the eligibility of Monarch-E, and a greater absolute benefit was observed in patients with high Ki-67 (37). Meanwhile, patients with high Ki-67 tend to have other combined highrisk features, so we believe that Ki-67 index alone may not serve as the decisive factor of using CDK 4/6 inhibitors. In addition, for patients with available genomic assays, those who have high genomic scores can also be classified as having high biological risk, and the opportunity of using CDK 4/6 inhibitors may be considered in this population in the future.

The median follow-up period of Monarch-E was approximately 19 months, which was shorter than PALLAS and Penelope B. We expect the results from long-term follow-up to confirm the status of adjuvant CDK4/6 inhibitor therapy in high-risk ER-positive patients.

\section{Multi-gene panel testing}

Are there postmenopausal patients with clinical presentations meeting the criteria of the MINDACT, TAILORx, RxPonder and similar trials with low-risk signatures and or recurrence scores (RS) $\leq 25$ who should receive chemotherapy? (I) No: 51.1\%; (II) Yes, if RS 21-25: 6.4\%; (III) Yes, if extensive LVI: 0.0\%; (IV) Yes, pT3pN1: $12.8 \%$; (V) Yes, if 3 positive LN: $8.5 \%$; (VI) Yes, pT3pN1 or 3 positive $\mathrm{LN}: 21.3 \%$ (abstain: 10 ).

For premenopausal women with node-negative breast cancers and recurrence score 16-25, or other lower-range genomic signature, the recommended treatment is: (I) Tam: $22.5 \%$; (II) OFS with Tam or AI: $53.1 \%$; (III) chemotherapy and endocrine therapy: $24.5 \%$ (abstain: 8).

Consider the experiences from ADAPT, MINDACT, TAILORX and RxPONDER, and other trials examining the role of endocrine therapy +/- chemotherapy in ERpositive HER2-negative breast cancer. For a patient with stage 3 (i.e., high anatomic stage), postmenopausal ER positive breast cancer. The preferred treatment is endocrine therapy and adjuvant chemotherapy (as opposed to endocrine therapy alone): (I) regardless of biomarkers: (i) Yes: 67.9\%; (ii) No: $32.1 \%$ (abstain: 0); (II) at very high stage such as if $\mathrm{N} 3(\geq 10+\mathrm{LN})$ or $\mathrm{T} 3 \mathrm{~N} 2$ : (i) Yes: $96.4 \%$; (ii) No: $3.6 \%$ (abstain: 1); (III) if grade 1 or 2 lobular breast cancer: (i) Yes: 48.0\%; (ii) No: 51.9\% (abstain: 4); (IV) if grade $1 /$ low grade/ Ki-67 <10\%: (i) Yes: $37.3 \%$; (ii) No: $62.8 \%$ (abstain: 5); (V) if recurrence score is $<11$ : (i) Yes: $34.0 \%$; (ii) No: 61.0\% (abstain: 8); (VI) if recurrence score is <25: (i) Yes: 58.3\%; (ii) No: 41.7\% (abstain: 9).

Multi-gene panel testing has become a useful tool in 
the selection of patients with ER-positive/HER2-negative breast cancer to receive de-escalation therapy. Meanwhile, according to the long-term outcome of MINDACT and TAILORx trial, the exploratory analyses indicated that the benefit of adding chemotherapy could be agedependent, and a clinically relevant benefit was observed in patients with low genomic risk yet younger than 50 years $(38,39)$. Therefore, the emission of chemotherapy in patients with low genomic risk should be handled with care. Chinese experts tend to add chemotherapy for ERpositive patients with positive lymph nodes unless the patient was postmenopausal, T1-3, and has low genomic risk. For premenopausal patients, we also prefer to add chemotherapy under most circumstances since a substantial proportion of premenopausal patients are likely to benefit from chemotherapy. For specific histological type and biomarkers such as $\mathrm{Ki}-67$, the consideration of endocrine responsiveness should be balanced with tumor burden and node metastases.

\section{Adjuvant therapy for HER2-positive breast cancer}

\section{Escalated anti-HER2 therapy}

Should patients with node-negative, HER2-positive breast cancers receive adjuvant pertuzumab (intravenous or subcutaneous formulation) in addition to trastuzumab? (I) Yes: 5.9\%; (II) No: 94.1\% (abstain: 6).

Should patients with HER2-positive breast cancers receive adjuvant neratinib after trastuzumab/pertuzumab as (neo)adjuvant therapy regimen, and/or trastuzumab emtansine-based therapy? (I) Yes: $1.9 \%$; (II) Yes, only if ER-positive: 5.8\%; (III) Yes, only if ER-positive and very high risk such as $\geq 4$ positive lymph nodes: $63.5 \%$; (IV) No: 28.9\% (abstain: 5).

In the section of adjuvant therapy for HER2-positive breast cancer, a topic worth discussion is the target population for escalated anti-HER2 therapy. Since no significant clinical benefit was observed in the nodenegative subgroup in APHINITY (15), a majority of experts agreed that the dual anti-HER2 blockade was not necessary in node-negative patients. The long-term follow-up data showed that adjuvant trastuzumab + chemotherapy (single HER2 blockade) was adequate for HER2-positive patients with negative lymph nodes and small tumor size. The APT trial showed the 7 -year RFI was $97.5 \%$ after wTH treatment (40). The 2-year DFS of early-stage patients also reached $97.8 \%$ when treated with $\mathrm{TC}+\mathrm{H}$ regimen (41).

Another escalated treatment strategy for high-risk
HER2-positive patients incorporating neratinib was investigated in the ExteNET trial (42). The prespecified subgroup analysis showed that the extended adjuvant therapy with neratinib for 1 year after trastuzumab and chemotherapy significantly enhanced iDFS in ERpositive patients with a tolerable safety profile (43). The combination of trastuzumab + pertuzumab has successfully reduced the early recurrence of high-risk patients, while further strategies are needed to reduce the long-term recurrence risk for ER-positive patients in view of the biological characteristics of ER-positive breast cancer. Therefore, most experts agreed that additional neratinib is a reasonable choice for ER-positive patients with very high risk even if the patient has received prior dual HER2 blockade therapy.

\section{Novel anti-HER2 strategies}

For women with stage 1, HER2 positive breast cancers, should trastuzumab emtansine be used instead of paclitaxel/ trastuzumab therapy? (I) Yes: 0.0\%; (II) No: 68.5\%; (III) Only under special circumstances: $31.5 \%$ (abstain: 2 ).

The phase II ATEMPT trial investigated T-DM1 $v s$. paclitaxel combined with trastuzumab $(\mathrm{T}+\mathrm{H})$ for stage I HER2-positive breast cancer in terms of toxicity and clinical benefit. The 3 -year DFS for patients receiving T-DM1 reached 97.5\% (95\% CI: 95.9-99.3\%), and T-DM1 was associated with less clinically relevant toxicities compared with $\mathrm{T}+\mathrm{H}$ (44), yet the trial did not meet the preplanned statistical endpoint. There lacks sufficient data supporting the regular use of T-DM1 in stage 1, HER2positive patients, while we also believe that some special conditions may justify the use of T-DM1, such as when cytotoxic chemotherapy is not acceptable for patients. It is worth noting that a meta-analysis involving 6,188 patients showed the incidence of T-DM1 induced thrombocytopenia was higher in Asian patients (45), so clinicians in China may be more cautious on the use of T-DM1, and careful surveillance is needed during the T-DM1 treatment.

\section{Adjuvant therapy for TNBC}

\section{Adjuvant immunotherapy}

Should patients with stage 2 or 3 TNBC, not treated in the neoadjuvant setting, but receiving adjuvant chemotherapy, also receive $\mathrm{PD}-1 / \mathrm{PD}-\mathrm{L} 1$ targeted treatment with an immune checkpoint inhibitor? (I) Yes: 9.6\%; (I) No: 90.4\% (abstain: 5).

For the use of immunotherapy in early-stage TNBC, 
we only have supporting evidence in neoadjuvant settings so far. Recent clinical trials such as KEYNOTE-522, IMPASSION031, and Geparneuvo (46) have revealed an augmented pCR after neoadjuvant therapy with ICIs, which indicated the potential of ICIs in the early-stage TNBC. Despite this, the use of neoadjuvant immunotherapy has not been widely acknowledged among Chinese experts due to the lack of data in Chinese patients and approval for indication. We believe that RFS and toxicity profile from long-term follow-up are needed, and predictive biomarkers are yet to be established. Several ongoing clinical trials are evaluating the use of ICIs in the adjuvant therapy for TNBC currently (NCT02826434, NCT03740893, NCT02954874, etc.), and we expect updates on results.

\section{PARP inhibitors}

Based on the tolerability of Olaparib in advanced, BRCA1/2 associated breast cancer, I would recommend adjuvant Olaparib in BRCA1/2 associated early-stage breast cancer if the OlympiA data show which of the following: (I) overall survival benefit, only: $7.7 \%$; (II) absolute iDFS at 3 years of $>10 \%: 25.0 \%$; (III) absolute iDFS at 3 years of $>5 \%: 48.1 \%$; (IV) absolute iDFS at 3 years of $>2 \%: 19.2 \%$ (abstain: 5 ).

For BRCA1/2-associated breast cancer patients, Olaparib has demonstrated significant improvement in PFS for metastatic patients $(47,48)$ and shown promising results in neoadjuvant settings (49). Based on the previous success, Olaparib has entered the arena of adjuvant therapy and has been under evaluation in the OlympiA trial. Since the aim of treating early breast cancer is to prevent recurrence, OlympiA sought to evaluate whether Olaparib can further reduce recurrence in germline $B R C A 1 / 2$-mutated patients with high risks.

According to the recently published data, the interim analysis of OlympiA demonstrated an absolute 3 -year iDFS of $8.8 \%$ in the Olaparib group $(85.9 \%$ vs. $77.1 \%$; HR: 0.58 ; 99.5\% CI: $0.41-0.82 ; \mathrm{P}<0.001)$, though the difference in OS between groups did not reach the interim-analysis boundary (50). This result was consistent with the most convincing answer among the panelist in St. Gallen BCC, and it promises to change the treatment landscape of early stage HER2-negative patients. Genetic testing of homologous repair mutations in early TNBC may help more patients gain benefits from this targeted therapy.

\section{Conclusions}

The $17^{\text {th }}$ ST. GALLEN International Breast Cancer
Consensus Conference focused on the controversies about the optimal primary treatment of early breast cancer. A great number of treatment recommendations were given. The expert recommendations of the $17^{\text {th }}$ ST. GALLEN conference could provide guidance to clinicians and the recommendations might suit the majority of patients in common clinical situations. However, clinicians should also aware that proper adjustments should be made according to the patient's socioeconomic status, complications, and tumor characteristics.

\section{Acknowledgments}

Funding: None.

\section{Footnote}

Conflicts of Interest: All authors have completed the ICMJE uniform disclosure form (available at https://dx.doi. org/10.21037/tbcr-21-7). JZ serves as the unpaid editorial board member of Translational Breast Cancer Research from May 2021 to April 2023. The other authors have no conflicts of interest to declare.

Etbical Statement: The authors are accountable for all aspects of the work in ensuring that questions related to the accuracy or integrity of any part of the work are appropriately investigated and resolved.

Open Access Statement: This is an Open Access article distributed in accordance with the Creative Commons Attribution-NonCommercial-NoDerivs 4.0 International License (CC BY-NC-ND 4.0), which permits the noncommercial replication and distribution of the article with the strict proviso that no changes or edits are made and the original work is properly cited (including links to both the formal publication through the relevant DOI and the license). See: https://creativecommons.org/licenses/by-nc-nd/4.0/.

\section{References}

1. Burstein HJ, Curigliano G, Thürlimann B, et al. CUSTOMIZING LOCAL AND SYSTEMIC THERAPIES FOR WOMEN WITH EARLY BREAST CANCER: The St. Gallen International Consensus Guidelines for treatment of early breast cancer 2021. Ann Oncol 2021. doi: 10.1016/j.annonc.2021.06.023. [Epub ahead of print]. 
2. Thomssen C, Balic M, Harbeck N, et al. St. Gallen/Vienna 2021: A Brief Summary of the Consensus Discussion on Customizing Therapies for Women with Early Breast Cancer. Breast Care (Basel) 2021;16:135-43.

3. Semiglazov VF, Semiglazov VV, Dashyan GA, et al. Phase 2 randomized trial of primary endocrine therapy versus chemotherapy in postmenopausal patients with estrogen receptor-positive breast cancer. Cancer 2007;110:244-54.

4. Spring LM, Gupta A, Reynolds KL, et al. Neoadjuvant Endocrine Therapy for Estrogen Receptor-Positive Breast Cancer: A Systematic Review and Meta-analysis. JAMA Oncol 2016;2:1477-86.

5. Iwata H, Masuda N, Yamamoto Y, et al. Validation of the 21-gene test as a predictor of clinical response to neoadjuvant hormonal therapy for $\mathrm{ER}+, \mathrm{HER} 2$-negative breast cancer: the TransNEOS study. Breast Cancer Res Treat 2019;173:123-33.

6. Ellis MJ, Suman VJ, Hoog J, et al. Randomized phase II neoadjuvant comparison between letrozole, anastrozole, and exemestane for postmenopausal women with estrogen receptor-rich stage 2 to 3 breast cancer: clinical and biomarker outcomes and predictive value of the baseline PAM50-based intrinsic subtype--ACOSOG Z1031. J Clin Oncol 2011;29:2342-9.

7. Korde LA, Somerfield MR, Carey LA, et al. Neoadjuvant Chemotherapy, Endocrine Therapy, and Targeted Therapy for Breast Cancer: ASCO Guideline. J Clin Oncol 2021;39:1485-505.

8. Ellis MJ, Tao Y, Luo J, et al. Outcome prediction for estrogen receptor-positive breast cancer based on postneoadjuvant endocrine therapy tumor characteristics. J Natl Cancer Inst 2008;100:1380-8.

9. Ellis MJ, Suman VJ, Hoog J, et al. Ki67 Proliferation Index as a Tool for Chemotherapy Decisions During and After Neoadjuvant Aromatase Inhibitor Treatment of Breast Cancer: Results From the American College of Surgeons Oncology Group Z1031 Trial (Alliance). J Clin Oncol 2017;35:1061-9.

10. Nitz U, Gluz O, Kreipe HH, et al. The run-in phase of the prospective WSG-ADAPT HR+/HER2trial demonstrates the feasibility of a study design combining static and dynamic biomarker assessments for individualized therapy in early breast cancer. Ther Adv Med Oncol 2020;12:1758835920973130.

11. van Ramshorst MS, van der Voort A, van Werkhoven ED, et al. Neoadjuvant chemotherapy with or without anthracyclines in the presence of dual HER2 blockade for HER2-positive breast cancer (TRAIN-2): a multicentre, open-label, randomised, phase 3 trial. Lancet Oncol 2018;19:1630-40.

12. van der Voort A, van Ramshorst MS, van Werkhoven ED, et al. Three-year follow-up of neoadjuvant chemotherapy with or without anthracyclines in the presence of dual HER2-blockade for HER2-positive breast cancer (TRAIN-2): A randomized phase III trial. J Clin Oncol 2020;38:501.

13. Schneeweiss A, Chia S, Hickish T, et al. Pertuzumab plus trastuzumab in combination with standard neoadjuvant anthracycline-containing and anthracycline-free chemotherapy regimens in patients with HER2-positive early breast cancer: a randomized phase II cardiac safety study (TRYPHAENA). Ann Oncol 2013;24:2278-84.

14. Huober J, Schneeweiss A, Blohmer JU, et al. Abstract P208-01: Factors predicting relapse in early breast cancer patients with a pathological complete response after neoadjuvant therapy - Results of a pooled analysis based on the GBG meta-database. Cancer Res 2019;79. doi: 10.1158/1538-7445.SABCS18-P2-08-01

15. Piccart M, Procter M, Fumagalli D, et al. Adjuvant Pertuzumab and Trastuzumab in Early HER2-positive Breast Cancer in the APHINITY Trial: 6 Years' FollowUp. J Clin Oncol 2021;39:1448-57.

16. Tolaney SM, Barry WT, Guo H, et al. Seven-year (yr) follow-up of adjuvant paclitaxel $(\mathrm{T})$ and trastuzumab $(\mathrm{H})$ (APT trial) for node-negative, HER2-positive breast cancer (BC). J Clin Oncol 2017;35:511.

17. Schmid P, Cortes J, Pusztai L, et al. Pembrolizumab for Early Triple-Negative Breast Cancer. N Engl J Med 2020;382:810-21.

18. Mittendorf EA, Zhang H, Barrios CH, et al. Neoadjuvant atezolizumab in combination with sequential nab-paclitaxel and anthracycline-based chemotherapy versus placebo and chemotherapy in patients with early-stage triple-negative breast cancer (IMpassion031): a randomised, double-blind, phase 3 trial. Lancet 2020;396:1090-100.

19. Masuda N, Lee SJ, Ohtani S, et al. Adjuvant Capecitabine for Breast Cancer after Preoperative Chemotherapy. N Engl J Med 2017;376:2147-59.

20. Li J, Yu K, Pang D, et al. Adjuvant Capecitabine With Docetaxel and Cyclophosphamide Plus Epirubicin for Triple-Negative Breast Cancer (CBCSG010): An OpenLabel, Randomized, Multicenter, Phase III Trial. J Clin Oncol 2020;38:1774-84.

21. Wang X, Wang SS, Huang H, et al. Effect of Capecitabine Maintenance Therapy Using Lower Dosage and Higher Frequency vs. Observation on Disease-Free Survival 
Among Patients With Early-Stage Triple-Negative Breast Cancer Who Had Received Standard Treatment: The SYSUCC-001 Randomized Clinical Trial. JAMA 2021;325:50-8.

22. Allison KH, Hammond MEH, Dowsett M, et al. Estrogen and Progesterone Receptor Testing in Breast Cancer: ASCO/CAP Guideline Update. J Clin Oncol 2020;38:1346-66.

23. Yi M, Huo L, Koenig KB, et al. Which threshold for ER positivity? a retrospective study based on 9639 patients. Ann Oncol 2014;25:1004-11.

24. Deyarmin B, Kane JL, Valente AL, et al. Effect of ASCO/ CAP guidelines for determining ER status on molecular subtype. Ann Surg Oncol 2013;20:87-93.

25. Fujii T, Kogawa T, Dong W, et al. Revisiting the definition of estrogen receptor positivity in HER2-negative primary breast cancer. Ann Oncol 2017;28:2420-8.

26. Wang S, Li J, Jiang Z, et al. Breast cancer patients with low estrogen receptor expression gain no significant survival benefit from endocrine therapy: a real-world study from China. Transl Breast Cancer Res 2020;1:14.

27. Ruhstaller T, Giobbie-Hurder A, Colleoni M, et al. Adjuvant Letrozole and Tamoxifen Alone or Sequentially for Postmenopausal Women With Hormone ReceptorPositive Breast Cancer: Long-Term Follow-Up of the BIG 1-98 Trial. J Clin Oncol 2019;37:105-14.

28. Mamounas EP, Bandos H, Lembersky BC, et al. Use of letrozole after aromatase inhibitor-based therapy in postmenopausal breast cancer (NRG Oncology/NSABP B-42): a randomised, double-blind, placebo-controlled, phase 3 trial. Lancet Oncol 2019;20:88-99.

29. Blok EJ, Kroep JR, Meershoek-Klein Kranenbarg E, et al. Optimal Duration of Extended Adjuvant Endocrine Therapy for Early Breast Cancer; Results of the IDEAL Trial (BOOG 2006-05). J Natl Cancer Inst 2018;110.

30. Gnant M, Steger G, Greil R, et al. Abstract GS3-01: A prospective randomized multi-center phase-III trial of additional 2 versus additional 5 years of anastrozole after initial 5 years of adjuvant endocrine therapy - results from 3,484 postmenopausal women in the ABCSG-16 trial. Cancer Res 2018;78. doi: 10.1158/1538-7445.SABCS17GS3-01. s

31. Fisher B, Dignam J, Bryant J, et al. Five versus more than five years of tamoxifen for lymph node-negative breast cancer: updated findings from the National Surgical Adjuvant Breast and Bowel Project B-14 randomized trial. J Natl Cancer Inst 2001;93:684-90.

32. Davies C, Pan H, Godwin J, et al. Long-term effects of continuing adjuvant tamoxifen to 10 years versus stopping at 5 years after diagnosis of oestrogen receptorpositive breast cancer: ATLAS, a randomised trial. Lancet 2013;381:805-16.

33. Gray RG, Rea D, Handley K, et al. aTTom (adjuvant Tamoxifen-To offer more?): Randomized trial of 10 versus 5 years of adjuvant tamoxifen among 6,934 women with estrogen receptor-positive $(\mathrm{ER}+)$ or ER untested breast cancer-Preliminary results. J Clin Oncol 2008;26:513.

34. Francis PA, Pagani O, Fleming GF, et al. Tailoring Adjuvant Endocrine Therapy for Premenopausal Breast Cancer. N Engl J Med 2018;379:122-37.

35. Johnston SRD, Harbeck N, Hegg R, et al. Abemaciclib Combined With Endocrine Therapy for the Adjuvant Treatment of HR+, HER2-, Node-Positive, HighRisk, Early Breast Cancer (monarchE). J Clin Oncol 2020;38:3987-98.

36. Mayer EL, Dueck AC, Martin M, et al. Palbociclib with adjuvant endocrine therapy in early breast cancer (PALLAS): interim analysis of a multicentre, open-label, randomised, phase 3 study. Lancet Oncol 2021;22:212-22.

37. O'Shaughnessy JA, Johnston S, Harbeck N, et al. Abstract GS1-01: Primary outcome analysis of invasive diseasefree survival for monarchE: abemaciclib combined with adjuvant endocrine therapy for high risk early breast cancer. Cancer Res 2021;81. doi: 10.1158/1538-7445. SABCS20-GS1-01.

38. Piccart M, van 't Veer LJ, Poncet C, et al. 70-gene signature as an aid for treatment decisions in early breast cancer: updated results of the phase 3 randomised MINDACT trial with an exploratory analysis by age. Lancet Oncol 2021;22:476-88.

39. Sparano JA, Gray RJ, Makower DF, et al. Adjuvant Chemotherapy Guided by a 21-Gene Expression Assay in Breast Cancer. N Engl J Med 2018;379:111-21.

40. Tolaney SM, Guo H, Pernas S, et al. Seven-Year FollowUp Analysis of Adjuvant Paclitaxel and Trastuzumab Trial for Node-Negative, Human Epidermal Growth Factor Receptor 2-Positive Breast Cancer. J Clin Oncol 2019;37:1868-75.

41. Jones SE, Collea R, Paul D, et al. Adjuvant docetaxel and cyclophosphamide plus trastuzumab in patients with HER2-amplified early stage breast cancer: a single-group, open-label, phase 2 study. Lancet Oncol 2013;14:1121-8.

42. Chan A, Delaloge S, Holmes FA, et al. Neratinib after trastuzumab-based adjuvant therapy in patients with HER2-positive breast cancer (ExteNET): a multicentre, 
randomised, double-blind, placebo-controlled, phase 3 trial. Lancet Oncol 2016;17:367-77.

43. Gnant M, Martin M, Holmes FA, et al. Abstract P2-13-01: Efficacy of neratinib in hormone receptor-positive patients who initiated treatment within 1 year of completing trastuzumab-based adjuvant therapy in HER2 + earlystage breast cancer: Subgroup analyses from the phase III ExteNET trial. Cancer Res 2019;79. doi: 10.1158/15387445.SABCS18-P2-13-01.

44. Tolaney SM, Trippa L, Barry W, et al. Abstract GS1-05: TBCRC 033: A randomized phase II study of adjuvant trastuzumab emtansine (T-DM1) vs. paclitaxel (T) in combination with trastuzumab $(\mathrm{H})$ for stage I HER2positive breast cancer (BC) (ATEMPT). Cancer Res 2020;80. doi: 10.1158/1538-7445.SABCS19-GS1-05.

45. Zhang J, Yang Y, Chen R, et al. Assessment of ethnic difference in the incidence of thrombocytopenia induced by trastuzumab emtansine (T-DM1): A meta-analysis. J Clin Oncol 2021;39:e15096.

46. Loibl S, Untch M, Burchardi N, et al. A randomised

doi: $10.21037 /$ tbcr-21-7

Cite this article as: Lin M, Jin Y, Zhang J. Expert review on systemic treatment in the St. Gallen International Breast Cancer Conference 2021. Transl Breast Cancer Res 2021;2:17. phase II study investigating durvalumab in addition to an anthracycline taxane-based neoadjuvant therapy in early triple-negative breast cancer: clinical results and biomarker analysis of GeparNuevo study. Ann Oncol 2019;30:1279-88.

47. Robson ME, Tung N, Conte P, et al. OlympiAD final overall survival and tolerability results: Olaparib versus chemotherapy treatment of physician's choice in patients with a germline BRCA mutation and HER2-negative metastatic breast cancer. Ann Oncol 2019;30:558-66.

48. Robson M, Im SA, Senkus E, et al. Olaparib for Metastatic Breast Cancer in Patients with a Germline BRCA Mutation. N Engl J Med 2017;377:523-33.

49. Eikesdal HP, Yndestad S, Elzawahry A, et al. Olaparib monotherapy as primary treatment in unselected triple negative breast cancer. Ann Oncol 2021;32:240-9.

50. Tutt ANJ, Garber JE, Kaufman B, et al. Adjuvant Olaparib for Patients with BRCA1- or BRCA2-Mutated Breast Cancer. N Engl J Med 2021;384:2394-405. 